\title{
Schistosomal peritonitis secondary to perforated appendicitis
}

Authors

Humphrey D Mazigo

Geofrey C Giiti²

Maria Zinga ${ }^{1}$

Jorg Heukelbach

Peter Rambau ${ }^{4}$

${ }^{1}$ Department of Medical

Parasitology and

Entomology, Weill-

Bugando University

College of Health Sciences,

Mwanza, Tanzania.

${ }^{2}$ Department of Surgery,

Bugando Medical Center,

Mwanza, Tanzania.

${ }^{3}$ Department of Community

Health, School of Medicine,

Federal University of Ceará,

Fortaleza, Brazil.

${ }^{4}$ Department of Pathology,

Weill-Bugando University

College of Health Sciences,

Mwanza, Tanzania.
Submitted on: 03/04/2010 Approved on: 03/20/2010

Correspondence to: Dr Humphrey D Mazigo Department of Medical Parasitology and

Entomology

Weill-Bugando University

College of Health

Sciences

P.O. Box 1464

Mwanza- Tanzania.

E-mail: umphreymazigo@

gmail.com/

humphreymazigo@

bugando.ac.tz

We declare no conflict of interest.

\begin{abstract}
In Tanzania, Schistosoma mansoni is endemic and causes intestinal schistosomiasis which affects various internal organs. However, worldwide there have been very few reports of cases of peritonitis due to schistosomal appendicitis. Here we report a rare case of schistosomal appendicitis with peritonitis in a 33 year-old male patient who recovered quickly after surgery.
\end{abstract}

Keywords: appendicitis; peritonitis; Schistosoma mansoni; Tanzania.

[Braz J Infect Dis 2010;14(6):628-630]@Elsevier Editora Ltda.

\section{INTRODUCTION}

Schistosomiasis causes chronic granulomatous disease affecting many systems and organs. The disease is endemic in many parts of the world with highest burden in Sub-Saharan Africa. In this region, $85 \%$ of the world population at risk is living, and about $97 \%$ of all infections occur. ${ }^{1,2}$ In Tanzania, both Schistosoma mansoni affecting the gastrointestinal system and $S$. haematobium affecting the urinary system, are endemic. ${ }^{1}$

Ectopic localizations of intestinal schistosomiasis have been described from almost all parts of the human body, and morbidity is usually caused by migrating ectopic eggs, such as in the female genital system and male prostate glands. ${ }^{4,5}$ The parasite is also known to affect multiple organs in the human host, including the appendix. ${ }^{3}$ Here we report a rare case of acute schistosomal peritonitis secondary to perforated appendix.

\section{CASE REPORT}

A 33 year-old male presented at Bugando Medical Centre (Mwanza region, Western Tanzania) with complains of intense abdominal pain that had started seven days earlier. He reported gradual onset of pain localized in right iliac fossa for the first three days, which became generalized. He experienced low grade fever accompanied by nausea and vomiting. The patient denied any obvious signs of constipation, diarrhea, melena, hematochezia, hematuria, dysuria, rashes, or joint symptoms.

Physical examination performed at hospital admission demonstrated that the patient was febrile $\left(39^{\circ} \mathrm{C}\right)$, not jaundiced and not pale. His blood pressure was $110 / 60 \mathrm{mmHg}$, pulse rate $78 \mathrm{bpm}$ and respiratory rate 18/ minute. The abdomen was not distended, silent with rebound tenderness and guarding. There was no obvious organomegaly. Other systems were essentially normal. Blood laboratory data showed hemoglobin $(\mathrm{Hb})$ concentration of $10.7 \mathrm{~g} / \mathrm{dL}$, leukocytosis, negative urinalysis, normal urea, electrolytes and liver function.

Radiological findings revealed gas under the diaphragm and multiple air fluid levels. A diagnostic hypothesis of peritonitis secondary to perforated intestine was made, and the patient was prepared for an emergency laparotomy.

During appendicectomy and abdominal lavage, he was noted to have an acutely inflamed appendix, foul smelling peritoneal fluid and matted bowels, and a perforated gangrenous appendix. The patient made an 
uneventful post operative recovery and was discharged after four days. Macroscopic examination of the appendix specimen showed its surface covered by fibropurulent materials, gangrenous and perforated. Histological evaluation was carried out on sections of paraffin blocks using the usual hematoxylin and eosin staining technique. Histopathological analysis showed acute purulent appendicitis characterized by infiltration of inflammatory cells, neutrophils, eosinophils (Figure 1) and numerous eggs of $S$. mansoni in the submucosal wall (Figure 2).

After histological report, stool and urine samples were collected and parasitological examination revealed eggs of $S$. mansoni in stool sample. The patient received a single dose of praziquantel $(40 \mathrm{mg} / \mathrm{kg})$, pending an outpatient review two weeks later, and recovered without any sequels.

Figure 1: Histological section of appendix(HE stain). Infiltration of inflammatory cells, neutrophils and eosinophils.

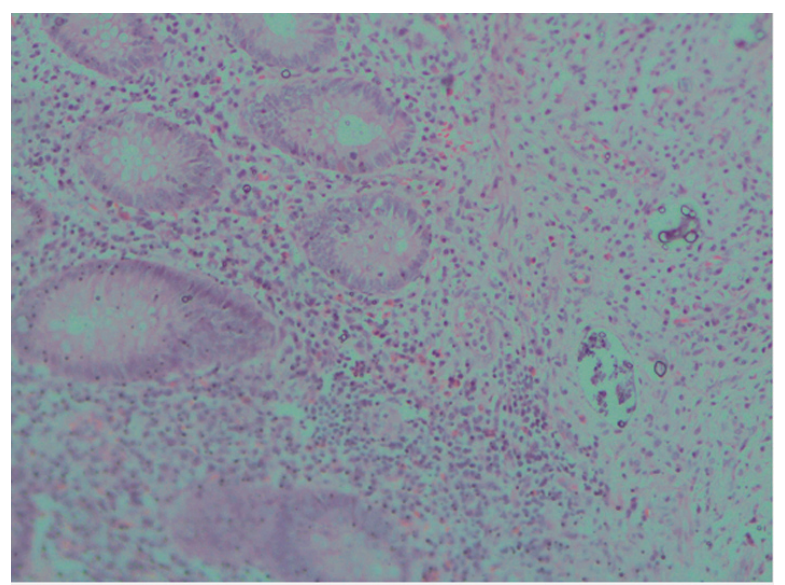

Figure 2: Histological section of appendix (HE stain). Numerous eggs of $S$. mansoni in the submucosal wall.

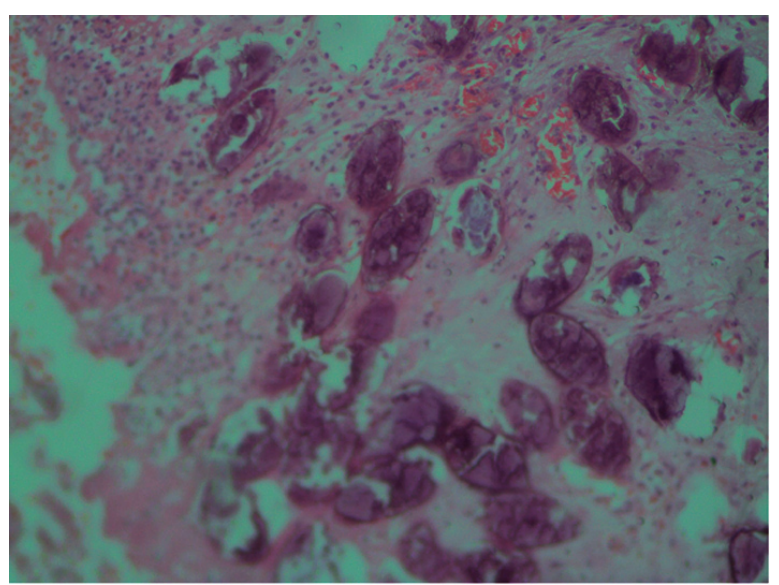

\section{DISCUSSION}

Here we report a rare case of acute peritonitis secondary to schistosomal appendicitis. Schistosomiasis of the appendix was first described by Turner in 1909.6 Since then, there have been reports of acute schistosomal granulamotous appendicitis from endemic populations in South America and Africa, as well as from travelers and migrants returning from endemic areas. ${ }^{3,7-12}$ In a retrospective study from Nigeria, in 27 (2.3\%) of more than 1,000 cases of appendicitis, schistosome eggs were seen in histological sections. ${ }^{8}$ About $56 \%$ of these cases were caused by S. mansoni, $26 \%$ by $S$. haematobium, and $19 \%$ by S. mansoni/S. haematobium coinfection. ${ }^{8}$ In another study from Nigeria, in $6.2 \%$ of appendicitis cases, chronic S. haematobium infection was evident. ${ }^{13}$ A more recent study also from Nigeria reported schistosomiasis of the appendix in $4.2 \%$ of surgical specimens. ${ }^{14}$

In contrast, peritoneal involvement in schistosomiasis seems to be a very rare event. Gali ${ }^{8}$ reported only one case of generalized peritonitis in 27 cases of schistosomal appendicitis. A recent case of schistosomal peritonitis with eggs found in the greater omentum and peritoneal cavity has been reported in an Egyptian migrant to Greece. ${ }^{15}$

The role of S. mansoni in the pathogenesis of appendicitis has long been debated and it is not fully understood if schistosomiasis of the appendix in fact induces acute appendicitis. One author hypothesized that ischemic changes due to egg emboli is responsible for reduced mucosal immunity, subsequently leading to bacterial infection. ${ }^{10}$ Others proposed that chronic schistosomal granulamotous inflammation, fibrosis, narrowing of the lumen and swelling of bowel wall may lead to secondary obstruction and acute appendicitis..$^{9,14}$

A critical appraisal of the association between schistosomiasis and acute appendicitis is reported from the histological findings characterized by infiltrations of the submucosal and the muscular layer by polymorphonuclear leukocytes, in addition to the presence of schistosome eggs. ${ }^{14}$ In the case of schistosomiasis mansoni, intramuscular oviposition (submucosa) may cause an obstructive type of appendicitis with a greater risk of perforation. ${ }^{3}$ Serosal involvement causes inflammation and the formation of adhesions. ${ }^{3}$

Confirmation of schistosomal involvement of organs is usually made by histological diagnosis, due to the absence of pathognomonic clinical or operative findings. Thus, a clear communication between surgeons and pathologists for management of patients with suspected appendicitis is required for proper management.

The presented case shows that intestinal schistosomiasis can cause acute peritonitis, which should be considered a differential diagnosis in patients presenting 
with features of appendicitis in schistosomiasis-endemic areas. In non-endemic areas, physicians need to be aware of these atypical presentations in migrant populations and travelers. Appendectomy specimens should be sent for histological review so that patients can benefit from full investigations and specific antiparasitic treatment.

\section{REFERENCES}

1. Steinmann P, Keiser J, Bos R, Tanner M, Utzinger J Schistosomiasis and water resources development: systematic review, meta-analysis, and estimates of people at risk. Lancet Inf Dis 2006; 6;411-25.

2. Gryseels B, Polman K, Clerinx J, Kestens L. Human schistosomiasis. Lancet 2006; 368;1106-18.

3. Adebamowo CA, Akang EEU, Ladipo JK, Ajao OG. Schistosomiasis of the appendix. Br J Surg 1991; 78:1219-12.

4. Bacelar A, Castro LG, de Queiroz AC, Café E. Association between prostate cancer and schistosomiasis in young patients: a case report and literature review. Braz J Infect Dis2007; 11:520-2.

5. King $\mathrm{CH}, \mathrm{Mahmoud}$ AAF. Schistosoma and other trematodes. In: Gorbarch SL, Bartlett JG, Blacklow NR, eds. Infectious Diseases. Philadelphia: WB. Saunders Company, 1998.
6. Halkic N, Abdelmoumene A, Gintzburger D, Mosimann F Schistosomal appendicitis in pregnancy. Swiss Surgery, 2002; 8;121-2.

7. Adehossi E, Parola P. Schistosomal appendicitis. Lancet Inf Dis 2004; 4:498.

8. Gali BM, Nggada HA, Eni EU. Schistosomiasis of the appendix in Maiduguri. Trop Doctor 2006; 36:162-3.

9. Nandipati K, Parithivel V, Niazi M. Schistosomiasis: a rare case of acute appendicitis in the African American population in the United States. Am Surg 2008; 74:221-3.

10. Terada T. Schistosomal appendicitis: Incidence in Japan and a case report. World J Gastroenterol 2009; 15:1648-9.

11. Webb JK, Thompson G. Schistosomal appendicitis in a Sudanese immigrant. Med J Aust 2009; 12:716-7.

12. Lambertucci JR, Santos Silva LC, Miranda D. Schistosomiasis mansoni of the appendix in a patient with acute appendicitis. Rev Soc Bras Med Trop 2008; 41:217-8.

13. Duvie SO, Diffang C, Guirguis MN. The effects of Schistosoma haematobium infestation on the vermiform appendix: the Nigerian experience. J Trop Med Hyg 1987; 90:13-8.

14. Badmos KB, Komolafe AO, Akintola L, Rotimi O. Schistosomiasis presenting as acute appendicitis. East Afr Med J 2006; 83:528-32.

15. Konstantinidou E, Alexiou C, Deminakou M, Sakellaridis T, Fotopoulos A, Antsaklis G. Schistosomal peritonitis: a rare cause of acute abdomen. Trans R Soc Trop Med Hyg 2009; 103:1068-70. 